\title{
EMBEDDING NIL ALGEBRAS IN TRAIN ALGEBRAS
}

\author{
by HENRIQUE GUZZO JR
}

(Received 4th November 1992)

Dedicated to the memory of Philip Holgate

\begin{abstract}
We generalize the classical example, due to Abraham, of a train algebra that is not special train, to non necessarily commutative right nil algebras of index $n$.
\end{abstract}

1980 Mathematics subject classification: 17 D92.

\section{Preliminaries}

In [1], Abraham gave the first example of a commutative train algebra which is not special triangular. A second example was given later by Holgate [5]. Basically Abraham used an example (due to Suttles [7]) of a commutative nil algebra $A$ of nil index 4 which is not nilpotent, to which he added a unity (resp. an idempotent $e$ such that $e a=\frac{1}{2} a$ ) thus obtaining a train algebra of rank 5 (resp. of rank 4) which is not special triangular. We intend in this note to explore further this idea, in the more general context of non commutative algebras of arbitrary right nil index $n$ and possibly infinite dimensional. We prove, in particular, that every right nil algebra of nil index $n$ can be embedded as the kernel of a right train algebra of rank $n$ or $n+1$. We should observe that every commutative nilpotent algebra $A$ of dimension $n$ can be embedded trivially in many ways in a special triangular, hence train, algebra. In fact, from the sequence $A \supseteq A^{2} \supseteq \cdots \supseteq A^{k-1} \supseteq A^{k}=0$ we obtain by the usual lifting process a basis $c_{1}, \ldots, c_{n}$ such that for $i<j, c_{i} c_{j}$ is a linear combination of $c_{j+1}, \ldots, c_{n}$. Then we add an indempotent $c_{0}$ such that $c_{0} A^{i} \subseteq A^{i}$ for all $i=1, \ldots, k$. This construction can be done also for non commutative nil algebras, thus yielding right or left train algebras.

We will omit henceforth the word "right". The case of left nil algebras is treated similarly. For basic facts about genetic algebra theory, the reader can consult [8] or [4].

Let $\mathbb{N}$ be the set of natural numbers and $F$ a field. We define recursively two functions from $\mathbb{N} \times \mathbb{N} \times F$ to $F$, denoted by $(n, j ; r)$ and $[n, j ; r]$, by:

(i) For $n<j,(n, j ; r)=0$; for $n \geqq j,(n, 0 ; r)=1$ and

$$
(n, j ; r)=(-1)^{j}\left(\left(\begin{array}{c}
n-1 \\
j
\end{array}\right) r^{j}+\left(\begin{array}{c}
n-1 \\
j-1
\end{array}\right) r^{j-1}\right) \text { if } j \geqq 1
$$


(ii) For $n<j,[n, j ; r]=0$; for $n \geqq j,[n, 0 ; r]=r^{1-\delta_{0 n}}$ and

$$
[n, j ; r]=\sum_{i=0}^{n-j}[n-1-i, j-1 ; r] r^{i} \quad \text { if } \quad j \geqq 1,
$$

where $\delta_{i j}$ is Kronecker's symbol. Observe that for $r=1,(n, j ; 1)=(-1)^{j}\left(\begin{array}{l}n \\ j\end{array}\right)$.

These functions satisfy the following identities, which are proved either by induction or either directly from the definition:

For all $n, j \in \mathbb{N}$ and $r \in F$, we have the equalities:

(a) $[n, n: r]=1$;

(b) $[n, n-1 ; r]=n r \quad(n \geqq 1)$;

(c) $(n, j ; r)=(n-1, j ; r)-(n-1, j-1 ; r) r \quad(n \geqq 2, j \geqq 1)$;

(d) $[n, j ; r]=[n-1, j ; r] r+[n-1, j-1 ; r] \quad(n \geqq 1, j \geqq 1)$.

In a similar way we prove that:
(e) $\sum_{j=0}^{k}(n, j ; r)=(-1)^{k}\left(\begin{array}{c}n-1 \\ k\end{array}\right) r^{k}$
$(0 \leqq k \leqq n-1) ;$
(f) $\sum_{j=0}^{n}(n, j ; r)=0$
$(n \geqq 1) ;$
(g) $\sum_{i=0}^{n-j}[n-i, j ; r](n-1, i ; r)=0$
$(0 \leqq j \leqq n-2)$
(h) $\sum_{i=1}^{n}(-1)^{i-1}(2 r-1)^{i-1}[n, i ; r]=1$
$(n \geqq 1)$.

Recall that a baric algebra over the field $F$ is an ordered pair $(A, \omega)$ where $A$ is any algebra over $F$ and $\omega: A \rightarrow F$ is a nonzero homomorphism. The set $N=\{x \in A: \omega(x)=0\}$ is a two sided ideal of $A$, of codimension 1 . If $c$ is any element of $A$ such that $\omega(c)=1$, we have a direct sum decomposition $A=F c \oplus N$, due to the equality $a=$ $\omega(a) c+(a-\omega(a) c)$, for all $a \in A$. If $A$ has an idempotent $e$ such that $\omega(e)=1$ (this happens for most of the relevant examples in the theory of genetic algebras) then $A=F e \oplus N$ so for all $b \in A, b=\omega(b) e+a$, where $a \in N$. For $b \in A$ let $b^{k}$, the (right) principal pqwer of $b$, be defined by $b^{1}=b, b^{k+1}=b^{k} b$ for $k \geqq 1$. When there are elements $\gamma_{1}, \ldots, \gamma_{n-1} \in F$ such that 


$$
b^{n}+\gamma_{1} \omega(b) b^{n-1}+\cdots+\gamma_{n-1} \omega(b)^{n-1} b=0, \text { for all } b \in A \text {, }
$$

$A$ will be called a principal train algebra. The rank of $(A, \omega)$ is the degree of the equation (1) of minimal degree satisfied by $(A, \omega)$. Our next proposition will enable us to treat the problem stated in the preliminaries. We remark that when $(A, \omega)$ satisfies $b^{n}+\gamma_{1} \omega(b) b^{n-1}+\cdots+\gamma_{n-1} \omega(b)^{n-1} b=0$ then necessarily $1+\gamma_{1}+\cdots+\gamma_{n-1}=0$. For this, apply $\omega$ to this identity, with $\omega(b)=1$.

Proposition 1. Suppose we are given a baric algebra $(A, \omega)$ with a central idempotent $e$ of weight 1 such that ea=ra $(=a e)$, for some fixed $r \in F$, for all $a \in N=\operatorname{ker} \omega$. Then for $a$ given $b=\omega(b) e+a$ and for given elements $\gamma_{0}=1, \gamma_{1}, \ldots, \gamma_{n-1} \in F$, we have

$$
b^{n}+\gamma_{1} \omega(b) b^{n-1}+\cdots+\gamma_{n-1} \omega(b)^{n-1} b=\left(\sum_{i=0}^{n-1} \gamma_{i}\right) \omega(b)^{n} e+\sum_{j=1}^{n}\left(\sum_{i=0}^{n-j}[n-i, j ; r] \gamma_{i}\right) \omega(b)^{n-j} a^{j}
$$

Proof (sketch). We proceed by induction, the case $n=2$ being trivial. Then

$$
\begin{aligned}
b^{n+1}+\gamma_{1} \omega(b) b^{n}+\cdots+\gamma_{n} \omega(b)^{n} b=\left[b^{n}+\gamma_{1} \omega(b) b^{n-1}+\cdots+\gamma_{n-1} \omega(b)^{n-1} b\right] b+\gamma_{n} \omega(b)^{n} b \\
=\left[\left(\sum_{i=0}^{n-1} \gamma_{i}\right) \omega(b)^{n} e+\sum_{j=1}^{n}\left(\sum_{i=0}^{n-j}[n-i, j ; r] \gamma_{i}\right) \omega(b)^{n-j} a^{j}\right](\omega(b) e+a)+\gamma_{n} \omega(b)^{n}(\omega(b) e+a) .
\end{aligned}
$$

The rest of the proof is obtained by combining the properties of the function $[n, j ; r]$ stated above. We omit the details, which are not interesting in their own.

The following lemma is well known:

Lemma. Let $A$ be a right nil algebra of nil index $s \geqq 2$. If $a \in A$ and $a^{s-1} \neq 0$, then $a$, $a^{2}, \ldots, a^{s-1}$ are linearly independent.

\section{The case $2 r \neq 1$}

Theorem 1. Let $(A, \omega)$ be a baric algebra, e $A$ a central idempotent of weight 1 such that ea $=r a(=a e)$ for all $a \in \operatorname{ker} \omega$. Suppose that $\operatorname{ker} \omega$ is a right nil algebra of nil index $s \geqq 2$. Then for every $b \in A$, we have $b^{s+1}+\gamma_{1} \omega(b) b^{s}+\cdots+\gamma_{s} \omega(b)^{s} b=0$ where $\gamma_{i}=(s, i ; r)$ for $i=0,1, \ldots, s$. Moreover, no other similar relation involving the powers $b, b^{2}, \ldots, b^{s}$ will hold and hence the rank of $A$ is $s+1$.

Proof. From Proposition 1, for all $b \in A, b=\omega(b) e+a, a \in \operatorname{ker} \omega$, we have

$$
b^{s+1}+\gamma_{1} \omega(b) b^{s}+\cdots+\gamma_{s} \omega(b)^{s} b=\sum_{j=1}^{s+1}\left(\sum_{i=0}^{s+1-j}[s+1-i, j ; r] \gamma_{i}\right) \omega(b)^{s+1-j} a^{j}
$$


because $1+\gamma_{1}+\cdots+\gamma_{s}=\sum_{i=0}^{s}(s, i ; r)=0$ by equation ( $\left.f\right)$ above. But in the above sum, $a^{s}=a^{s+1}=0$ so that

$$
b^{s+1}+\gamma_{1} \omega(b) b^{s}+\cdots+\gamma_{s} \omega(b)^{s} b=\sum_{j=1}^{s-1}\left(\sum_{i=0}^{s+1-j}[s+1-i, j ; r] \gamma_{i}\right) \omega(b)^{s+1-j} a^{j}
$$

From equation $(\mathrm{g})$, we have

$$
\sum_{i=0}^{s+1-j}[s+1-i, j ; r] \gamma_{i}=\sum_{i=0}^{s+1-j}[s+1-i, j ; r](s, i ; r)=0 \text { for } 1 \leqq j \leqq s-1
$$

This shows that $(A, \omega)$ is a train algebra of rank $\leqq s+1$. We show that $s+1$ is a minimal degree. Suppose, to the contrary, that for some $t \leqq s+1$ and constants $\alpha_{1}, \ldots, \alpha_{t-1}$ in $F$ we have $b^{t}+\alpha_{1} \omega(b) b^{t-1}+\cdots+\alpha_{t-1} \omega(b)^{t-1} b=0$ for all $b \in A$. In particular, when $\omega(b)=0$, we have $b^{t}=0$ and so $s \leqq t$. We are left with two possibilities: $t=s$ or $t=s+1$. Suppose we have $t=s$. Choose $a \in \operatorname{ker} \omega$ with $a^{s-1} \neq 0$ so that $a, a^{2}, \ldots, a^{s-1}$ are linearly independent. Apply to the element $b=e+a$ the equality in Proposition 1 to get

$$
\begin{gathered}
0=b^{s}+\alpha_{1} b^{s-1}+\cdots+\alpha_{s-1} b=\sum_{j=1}^{s}\left(\sum_{i=0}^{s-j}[s-i, j ; r] \alpha_{i}\right) a^{j} \\
=\sum_{j=1}^{s-1}\left(\sum_{i=0}^{s-j}[s-i, j: r] \alpha_{i}\right) a^{j} \quad \text { as } \quad a^{s}=0
\end{gathered}
$$

We have now a triangular system of linear equations in the unknowns $\alpha_{i}$ :

$$
\sum_{i=0}^{s-j}[s-i, j ; r] \alpha_{i}=0, \quad 1 \leqq j \leqq s-1 .
$$

Multiply the $j$ th equation by $(-1)^{j-1}(2 r-1)^{j-1}$ and sum up all the resulting equations to get

$$
\sum_{j=1}^{s-1}\left(\sum_{i=0}^{s-j}(-1)^{j-1}(2 r-1)^{j-1}[s-i, j ; r] \alpha_{i}\right)=0
$$

Reordering the summands, we get

$$
\sum_{i=1}^{s-1}\left(\sum_{j=1}^{s-i}(-1)^{j-1}(2 r-1)^{j-1}[s-i, j ; r]\right) \alpha_{i}+\sum_{j=1}^{s-1}(-1)^{j-1}(2 r-1)^{j-1}[s, j ; r]=0 .
$$


By equation (h), we have

$$
0=-(-1)^{s-1}(2 r-1)^{s-1}[s, s ; r]+1+\alpha_{1}+\cdots+\alpha_{s-1}=(-1)^{s}(2 r-1)^{s-1}[s, s ; r],
$$

and by equation (a), we have $[s, s ; r]=1$ and so $2 r=1$, a contradiction. We must have then $t=s+1$, which proves that the rank of $A$ is $s+1$.

Corollary 1. (Abraham [1]) Suppose $(A, \omega)$ is a baric algebra with a unity element $1_{A}$ and $\operatorname{ker} \omega$ is nil of nil index $s \geqq 2$. Then $(A, \omega)$ is a train algebra of rank $s+1$, satisfying the equation $\left(x-\omega(x) 1_{A}\right)^{s} x=0$.

Proof. Our theorem, with $e=1_{A}$ and $r=1$, says that $(A, \omega)$ is a train algebra of rank $s+1$ and the coefficients $\gamma_{i}$ of its train equation are given by:

$$
\gamma_{i}=(s, i ; 1)=(-1)^{i}\left(\left(\begin{array}{c}
s-1 \\
i
\end{array}\right)+\left(\begin{array}{c}
s-1 \\
i-1
\end{array}\right)\right)=(-1)^{i}\left(\begin{array}{c}
s \\
i
\end{array}\right), \quad \text { for } \quad 0 \leqq i \leqq s .
$$

Then for every $b \in A$,

$$
\begin{gathered}
0=b^{s+1}+\gamma_{1} \omega(b) b^{s}+\cdots+\gamma_{s} \omega(b)^{s} b \\
=\left(b^{s}+\left(\begin{array}{l}
s \\
1
\end{array}\right)(-\omega(b)) b^{s-1}+\cdots+\left(\begin{array}{c}
s \\
s-1
\end{array}\right)(-\omega(b))^{s-1}+(-\omega(b))^{s} 1_{A}\right) b \\
=\left(b-\omega(b) 1_{A}\right)^{s} b .
\end{gathered}
$$

Given any algebra $N$ over the field $F$ and a linear mapping $\tau: N \rightarrow N$, we can obtain a baric algebra with a central idempotent of weight 1 in the following way (see [2] for details): take $A=F e \oplus N$ and the product and a weight function $\omega$ given by

$$
(\alpha, a)(\beta, b)=(\alpha \beta, a b+\tau(\alpha b+\beta a)) ; \quad \omega(\alpha, a)=\alpha,
$$

where $\alpha, \beta \in F ; a, b \in N$. Then $(1,0)=e$ satisfies the above conditions. If $N$ is nil of nil index $s$ and $\tau$ is the linear mapping $h_{r}: a \mapsto r a$, where $2 r \neq 1, r \in F$, then the resulting baric algebra satisfies the equation of Theorem 1 . We denote this algebra by $\left[N, h_{r}\right]$.

Corollary 2. Suppose $M$ and $N$ are two nil algebras of nil indices $m$ and $n$ respectively, $m, n \geqq 2$. Suppose $r, s \in F$ satisfy $2 r \neq 1$ and $2 s \neq 1$. Then $\left[M, h_{r}\right]$ and $\left[N, h_{s}\right]$ are isomorphic (as baric algebras) if and only if $M$ and $N$ are isomorphic and $r=s$.

Proof. Suppose $\left[M, h_{r}\right]$ and $\left[N, h_{s}\right]$ are isomorphic, let $\phi$ be an isomorphism. By [2, Proposition 1 and Corollary] there exists an isomorphism $\theta: M \rightarrow N$ and $c \in N$ such that $\phi(\alpha, a)=(\alpha, \alpha c+\theta(a)), c^{2}+2 s c=c$ and the diagram 


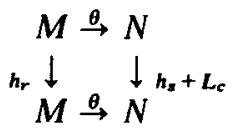

is commutative. But $c^{2}+2 s c=c$ implies $c^{n}=(1-2 s)^{n-1} c$ and so $c=0$. From the diagram, for all $a \in M, r \theta(a)=s \theta(a)$ with means necessarily $r=s$.

The proof of the converse is trivial.

\section{The case $r=\frac{1}{2}$}

We assume now the characteristic of $F$ is not 2 . The following theorem is a generalization of Abraham's example in [1].

Theorem 1'. Suppose $(A, \omega)$ is a baric algebra, $e \in A$ is a central idempotent of weight 1 such that $e a=\frac{1}{2} a(=a e)$. Suppose that $N=\operatorname{ker} \omega$ is nil of nil index $s \geqq 2$. Then for every $b \in A$, we have $b^{s}+\gamma_{1} \omega(b) b^{s-1}+\cdots+\gamma_{s-1} \omega(b)^{s-1} b=0$ where $\gamma_{i}=\left(s-1, i ; \frac{1}{2}\right)$ for $i=0,1, \ldots, s-1$. Moreover, no other similar relation involving the powers $b, \ldots, b^{s-1}$ will hold and so the rank of $A$ is $s$.

Proof. For $b=\omega(b) e+a, b \in A$ and $a \in \operatorname{ker} \omega$, we have, as in the proof of Theorem 1,

$$
b^{s}+\gamma_{1} \omega(b) b^{s-1}+\cdots+\gamma_{s-1} \omega(b)^{s-1} b=\sum_{j=1}^{s-1}\left(\sum_{i=0}^{s-j}\left[s-i, j ; \frac{1}{2}\right] \gamma_{i}\right) \omega(b)^{s-j} a^{j} \text { as } a^{s}=0
$$

From equation (f),

$$
\sum_{i=0}^{s-j}\left[s-i, j ; \frac{1}{2}\right] \gamma_{i}=\sum_{i=0}^{s-j}\left[s-i, j ; \frac{1}{2}\right]\left(s-1, i ; \frac{1}{2}\right)=0, \quad 1 \leqq j \leqq s-2 .
$$

When $j=s-1$, we have,

$$
\begin{gathered}
\sum_{i=0}^{1}\left[s-i, s-1 ; \frac{1}{2}\right]\left(s-1, i ; \frac{1}{2}\right)=\left[s, s-1 ; \frac{1}{2}\right]\left(s-1,0 ; \frac{1}{2}\right)+\left[s-1, s-1 ; \frac{1}{2}\right]\left(s-1,1 ; \frac{1}{2}\right) \\
=\frac{s}{2}+(-1)\left(\left(\begin{array}{c}
s-2 \\
1
\end{array}\right) \frac{1}{2}+\left(\begin{array}{c}
s-2 \\
0
\end{array}\right)\right)=0 .
\end{gathered}
$$

This proves that $b^{s}+\gamma_{1} \omega(b)^{s-1}+\cdots+\gamma_{s-1} \omega(b)^{s-1} b=0$ for all $b \in A$. No other similar relation, involving only $b^{s-1}, \ldots, b$ can hold because this would imply that all elements $b$ of weight 0 would satisfy $b^{s-1}=0$, a contradiction to the nil index of ker $\omega$. 
It is obvious that if $N$ is nil algebra of nil index $s$, then $\left[N, h_{1 / 2}\right]$ satisfies the equation of Theorem 1'.

We can easily prove the following:

Corollary. Suppose $M$ and $N$ are $F$-algebras. Then $\left[M, h_{1 / 2}\right]$ and $\left[N, h_{1 / 2}\right]$ are isomorphic (as baric algebras) if and only if $M$ and $N$ are isomorphic.

\section{Complements}

In this paragraph, we collect some properties of the algebras $\left[M, h_{r}\right]$. For any baric algebra $(A, \omega)$, the ideal generated by all the elements $x^{2}-\omega(x), x \in A$ is called the Etherington's ideal of $A$. For several classes of baric algebras, we can describe more explicitly this ideal, see, for instance, [7, Lemma 9.19] for Bernstein algebras.

Proposition 2. For a given algebra $M$ over $F$, the Etherington's ideal of $\left[M, h_{r}\right]$ is $M$ when $r \neq \frac{1}{2}$ and is the ideal generated by the squares of elements of $M$, when $r=\frac{1}{2}$.

Proof. For $b=\omega(b) e+a$ (notations as above), we have $b^{2}-\omega(b) b=a^{2}+\omega(b)(2 r-1) a$. When $r=\frac{1}{2}$, we have the desired result. When $r \neq \frac{1}{2}$, we have $b^{2}-\omega(b) b-a^{2}=$ $\omega(b)(2 r-1) a$ so $a$ belongs to the above ideal, that is, $M$ is contained in the Etherington's ideal. The converse inclusion is obvious.

In [2], the authors have introduced the concept of decomposable baric algebra. It means that $N=\operatorname{ker} \omega$ can be decomposed as a direct sum of two nonzero two sided ideals of the baric algebra, contained in $N$. In our case, we have obviously the following result.

Proposition 3. $\left[M, h_{r}\right]$ is decomposable if and only if $M$ is decomposable as a direct sum $M=M_{1} \oplus M_{2}$ where $M_{i}$ are nonzero two sided ideals of $M$.

Remarks. Suppose $(A, \omega)$ is a baric algebra having a central idempotent $e$ of weight 1 such that $e a=r a(=a e)$ for all $a \in \operatorname{ker} \omega$, for some $2 r \neq 1, r \in F$. Suppose moreover that $\operatorname{ker} \omega$ is nil of nil index $s \geqq 2$. Let $e^{\prime}$ be another idempotent of weight 1 , say $e^{\prime}=e+c$, $c \in \operatorname{ker} \omega$. Then $e+c=e^{\prime}=e^{\prime 2}=e+2 c e+c^{2}=e+2 r c+c^{2}$, so $c^{2}=(1-2 r) c$. From this we have $c^{k}=(1-2 r)^{k-1} c$ for $k \geqq 2$. By the nil property, $0=c^{s}=(1-2 r)^{s-1} c$ so $c=0$, and $A$ has a unique idempotent of weight 1 .

In the case $r=\frac{1}{2}$, we may have many idempotents; moreover, for another idempotent $e^{\prime}$, it may happen that $e^{\prime} a \neq \frac{1}{2} a$. For an example, go back to Abraham's example in [1] and [5]. It is easy to see that $e_{0}=c_{0}+c_{1}$ is an idempotent of weight 1 and $e_{0} c_{3}=\frac{1}{2} c_{3}+c_{4}$.

\section{REFERENCES}

1. V. M. Abraham, A note on train algebras, Proc. Edinburgh Math. Soc. 20(2) (1976), 53-58. 
2. R. Costa and H. Guzzo JR, Indecomposable baric algebras, Linear Algebra Appl. 183 (1993), 223-236.

3. R. Costa and H. Guzzo $\mathrm{J}_{\mathrm{R}}$, Indecomposable baric algebras, II, Linear Algebra Appl., to appear.

4. I. M. H. Etherington, Genetic Algebras, Proc. Roy. Soc. Edinburgh 59 (1939), 242-258.

5. P. Holgate, A train algebra that is not special triangular, Arch. Math. 50 (1988), 122-124.

6. R. D. Schafer, An introduction to nonassociative algebras (Academic Press, New York, 1966).

7. D. Suttres, A counterexample to a conjecture of Albert, Notices Amer. Math. Soc. A19 (1972), 566.

8. A. Wörz, Algebras in Genetics (Lecture Notes in Biomathematics 36, Springer, BerlinHeidelberg-New York, 1980).

Instituto de Matemática e Estatistica

Universidade de São Paulo

Caixa Postal 20570

01452-990-Sáo Paulo, Brazil

E-mail: guzzo@ime.usp.br 